\title{
MicroRNA-134-3p inhibits ovarian cancer progression by targeting flap structure-specific endonuclease 1 in vitro
}

\author{
MENGSHI ZHAO ${ }^{1}$, HONGJIE JI ${ }^{1}$, QIANG FU ${ }^{1}$, QIAN CHENG ${ }^{1}$, YU ZHANG ${ }^{2}$ and YU YANG ${ }^{1,3}$ \\ ${ }^{1}$ School of Minerals Processing and Bioengineering, Central South University, Changsha, Hunan 410083; \\ ${ }^{2}$ Department of Obstetrics and Gynecology, Xiangya Hospital, Central South University, Changsha, Hunan 410008; \\ ${ }^{3}$ Key Laboratory of Biometallurgy, Ministry of Education, Changsha, Hunan 410083, P.R. China
}

Received May 15, 2020; Accepted October 6, 2020

DOI: $10.3892 / o r .2020 .7844$

\begin{abstract}
Ovarian cancer (OC) is one of the most lethal gynecological malignancies in the world. The aim of the present study was to examine the role of microRNA (miR)-134-3p in OC. Reverse transcription-quantitative PCR was used to measure the expression levels of miR-134-3p. Cell Counting Kit-8, TUNEL, flow cytometric and colony formation assays were performed to examine the effects of miR-134-3p on OC cell proliferation. Moreover, wound healing and Transwell assays were performed to examine the effects on migration and invasion. In addition, western blot analyses were used to assess protein expression. Finally, the target genes of miR-134-3p were analyzed by bioinformatics analysis and Dual-Luciferase reporter assay. The results revealed that miR-134-3p expression was low in OC cells compared with in normal ovarian cells. The overexpression of miR-134-3p decreased cell viability, facilitated cell apoptosis, inhibited cell proliferation and arrested the cell cycle in SKOV-3 and OVCAR-3 cells. Furthermore, transfection using a miR-134-3p mimic inhibited the migration and invasion of SKOV-3 and OVCAR-3 cells, and decreased the protein expression levels of cyclooxygenase-2, matrix metalloproteinase (MMP)2 and MMP9. Bioinformatics analysis indicated that one of the potential target genes of miR-134-3p was flap structure-specific endonuclease 1 (FEN1), which was confirmed by dual-luciferase reporter assay. Moreover, overexpression of miR-134-3p decreased the expression levels of FEN1 in SKOV-3 and OVCAR-3 cells. Additionally, overexpression of FEN1 reversed the effects of the miR-134-3p mimic on the proliferation, migration and invasion of SKOV-3 and OVCAR-3 cells. Overall, the findings of the present study demonstrated that miR-134-3p may inhibit OC cell proliferation, migration and invasion by directly targeting FEN1.
\end{abstract}

Correspondence to: Professor Yu Yang, School of Minerals Processing and Bioengineering, Central South University, Changsha, Hunan 410083, P.R. China

E-mail: csuyangyu@csu.edu.cn

Key words: microRNA-134-3p, flap structure-specific endonuclease 1 , human ovarian cancer

\section{Introduction}

Ovarian cancer (OC) is one of the most lethal types of malignant gynecological cancer in the world (1). In 2018 in the United States, there were 22,240 new cases of OC and 14,070 OC-associated mortalities (2). In China, the latest reported number of OC-associated mortalities was 25,000 in 2015 (3). Although surgery, chemotherapy and radiotherapy have made marked progresses in the treatment of $\mathrm{OC}$, the treatment of patients with advanced OC with distant metastasis or recurrence remains a challenge, and the median overall survival (OS) time of patients with metastatic disease has not improved over the past decades (4). Therefore, the elucidation of the underlying mechanisms of $\mathrm{OC}$ and the identification of novel therapeutic targets to improve the clinical outcome of patients suffering from OC is of utmost importance.

MicroRNAs (miRNAs or miRs) are a class of RNAs containing 22 nucleotides that cannot be translated into proteins (5). miRNAs can act as suppressors of gene expression by binding to the 3 '-untranslated region (3'-UTR) of target mRNAs $(5,6)$. Previous studies have demonstrated that miRNAs serve vital roles in the progression of various types of cancer, such as ovarian, lung and breast cancer, by regulating the expression of genes involved in cell proliferation, apoptosis, differentiation and migration (7). For example, miR-15a-3p suppresses the proliferation and invasion of ovarian cancer cells by targeting Twist1 (8). This suggests the possible application of miRNAs in cancer diagnosis, treatment or prognosis $(9,10)$. Increasing evidence has indicated that miR-134 acts as a tumor suppressor by blocking the proliferation of various cancer cells through different signaling pathways $(11,12)$. For instance, miR-134 can regulate the activation of the PI3K signaling pathway to inhibit the proliferative and invasive activities of glioma cells and facilitate their apoptosis (13). Furthermore, miR-134 is mediated by interferon regulatory factor 1 to suppress the tumorigenesis and progression of osteosarcoma by targeting VEGFA and MYCN (14).

Flap structure-specific endonuclease 1 (FEN1) is a member of the Rad2 structure-specific nuclease family, involved in a number of DNA processing pathways to maintain genome stability $(15,16)$. For instance, through the interaction with proliferating cell nuclear antigen (PCNA), FEN1 helps coordinate Okazaki fragment maturation by removing RNA-DNA 
primers (17). FEN1 expression is upregulated in a number of types of cancer, such as cancer of the testes, lung and brain (18). In addition, accumulating evidence has indicated that miRNAs can inhibit FEN1 expression by directly binding to its 3'-UTR, leading to impaired DNA repair and repressed cancer progression $(19,20)$.

The present study aimed to reveal the role of miR-134-3p in $\mathrm{OC}$ by measuring the expression levels of miR-134-3p in OC cell lines. Cell Counting Kit (CCK)-8, TUNEL, flow cytometry, colony formation, wound healing and Transwell assays were used to assess the inhibition of miR-134-3p on OC cell proliferation, migration and invasion.

\section{Materials and methods}

Cell lines and cell culture. The human OC SKOV-3, A2780 and OVCAR-3 cell lines, and the normal ovarian epithelial ISOE-80 cell line were purchased from the American Type Culture Collection. A2780 and OVCAR-3 cells were maintained in DMEM supplemented with $10 \%$ fetal bovine serum (FBS) (both Gibco; Thermo Fisher Scientific, Inc.), $100 \mathrm{U} / \mathrm{ml}$ penicillin and $100 \mathrm{mg} / \mathrm{ml}$ streptomycin (HyClone; Cytiva). ISOE-80 cells were maintained in RPMI-1640 medium (Gibco; Thermo Fisher Scientific, Inc.) supplemented with $10 \%$ FBS, $100 \mathrm{U} / \mathrm{ml}$ penicillin and $100 \mathrm{mg} / \mathrm{ml}$ streptomycin. SKOV-3 cells were cultured in McCoy's 5A medium (Gibco; Thermo Fisher Scientific, Inc.) supplemented with 10\% FBS, $100 \mathrm{U} / \mathrm{ml}$ penicillin and $100 \mathrm{mg} / \mathrm{ml}$ streptomycin. All cells were cultured at $37^{\circ} \mathrm{C}$ with $5 \% \mathrm{CO}_{2}$.

Cell transfection. The chemically synthesized miR-134-3p mimic (miRBase, MIMAT0026481; 5'-CCUGUGGGC CACCUAGUCACCAA-3'), non-targeting mimic negative control (NC; 5'-UUCUCCGAACGUGUCACGUTT-3'), Lv-FEN1 and Lv-NC (expression vector, pcDNA3.1) were designed and obtained from Shanghai GenePharma Co., Ltd. Cell transfection was performed in SKOV-3 and OVCAR-3 cells using the cell electroporation system operator H1 (Etta Biotech Co. Ltd.) according to the manufacturer's protocol. Briefly, SKOV-3 and OVCAR-3 cells were collected and re-suspended in the electroporation buffer. The cell concentration was adjusted to $6 \times 10^{5}$ cells $/ \mathrm{ml}$ and mixed with miRNA mimics $(125 \mathrm{nM})$ and/or plasmid $(1 \mathrm{mg} / \mathrm{ml})$ prior to electroporation. A total of $100 \mu \mathrm{l}$ cell suspension mixed with RNAs was then added into a 96-well plate, and used for gene transfection using the operator $\mathrm{H} 1$. The electroporation device was operated at DC square wave with $200 \mathrm{~V}$ voltage, $100 \mu \mathrm{sec}$ duration, 2-sec interval and 6 pulses. After electroporation, the cells were diluted to appropriate concentrations and seeded into appropriate cell culture plates for use in further assays.

Reverse transcription-quantitative PCR (RT-qPCR). TRIzol ${ }^{\circledR}$ reagent (Invitrogen; Thermo Fisher Scientific, Inc.) was used for the isolation of total RNA from cells. Reverse transcription was performed using the 4X Reverse Transcription Master Mix kit (EZBioscience) according to the manufacturer's protocol. qPCR was performed on cDNA using FastStart Universal SYBR-Green Master Mix (Roche Diagnostics $\mathrm{GmbH}$ ). Briefly, the reaction mixture contained $25 \mu \mathrm{l}$ SYBRGreen Master Mix, $0.5 \mu \mathrm{l}$ each primer $(30 \mu \mathrm{mol} / \mathrm{l}), 5 \mu \mathrm{l}$ DNA template $(10 \mathrm{ng} / \mu \mathrm{l})$ and molecular-grade $\mathrm{H}_{2} \mathrm{O}$ to a final reaction volume of $45 \mu \mathrm{l}$. The amplification protocol consisted of one cycle of initial denaturation at $95^{\circ} \mathrm{C}$ for $5 \mathrm{~min}, 40$ cycles of denaturation at $95^{\circ} \mathrm{C}$ for $15 \mathrm{sec}$ and annealing/extension at $60^{\circ} \mathrm{C}$ for $1 \mathrm{~min}$. Gene expression levels were quantified using the $2^{-\Delta \Delta C \mathrm{q}}$ method (21). U6 and $\beta$-actin were used to normalize miR-134-3p or FEN1 expression, respectively. The PCR primer sequences for the selected genes were as follows: FEN1 forward, 5'-GTGAAGGCTGGCAAAGTCTA-3' and reverse, 5'-GTGAAGGCTGGCAAAGTCTA-3'; $\beta$-actin forward, 5'-GCTCGTCGTCGACAACGGCTC-3' and reverse, 5'-CAAACATGATCTGGGTCATCTTCTC-3'; miR-134-3p forward, 5'-CTGTGGGCCACCTAGTCACCAA-3' and reverse, 5'-GCTGTCAACGATACGCTACCTA-3'; U6 forward, 5'-CTCGCTTCGGCAGCACA-3' and reverse, 5'-AA CGCTTCACGAATTTGCGT-3'.

Cell proliferation assay. The transfected SKOV-3 and OVCAR-3 cells were seeded in 96-well plates at a density of $5 \times 10^{3}$ cells/well. The CCK-8 (Beyotime Institute of Biotechnology) colorimetric assay was performed to measure cell proliferation according to the manufacturer's protocol. Briefly, the supernatant was removed after 24,48 and $72 \mathrm{~h}$ of culture at $37^{\circ} \mathrm{C}$ and $5 \% \mathrm{CO}_{2}$. Subsequently, $100 \mu \mathrm{l}$ of respective medium containing $10 \mu \mathrm{l}$ CCK- 8 reagent was added to each well for $3 \mathrm{~h}$ at $37^{\circ} \mathrm{C}$. The absorbance was measured at $450 \mathrm{~nm}$ using a plate reader (Thermo Multiskan MK3 spectrophotometer; Thermo Fisher Scientific, Inc.). The optical density value was determined and used to construct a growth curve to assess cell proliferation.

Colony formation assay. SKOV-3 and OVCAR-3 transfected cells were seeded in $10-\mathrm{cm}$ dishes $\left(1 \times 10^{3}\right.$ cells/plate $)$ and treated as follows: First, the cells were cultured in complete culture medium for 21 days; subsequently, they were fixed in $4 \%$ paraformaldehyde for $30 \mathrm{~min}$ at $4^{\circ} \mathrm{C}$ and stained with Giemsa dye for $20 \mathrm{~min}$ at $4^{\circ} \mathrm{C}$. Images of cells were captured using a light microscope (magnification, $\mathrm{x} 40$ ), and the number of colonies, consisting of $\geq 3$ cells, was calculated.

TUNEL assay. The transfected SKOV-3 and OVCAR-3 cells were cultured for $\sim 48 \mathrm{~h}$ at $37^{\circ} \mathrm{C}$ and $5 \% \mathrm{CO}_{2}$. The apoptosis of the transfected cells was then detected by TUNEL assay as recommended in the ApopTag ${ }^{\circledR}$ Plus Peroxidase in Situ Apoptosis kit (Sigma-Aldrich; Merck KGaA). Briefly, the cells were fixed with $4 \%$ paraformaldehyde for $30 \mathrm{~min}$ at room temperature, and permeabilized with $0.1 \%$ Triton X-100 in $0.1 \%$ sodium citrate for $2 \mathrm{~min}$ at $4^{\circ} \mathrm{C}$. Subsequently, the cells were incubated with $1 \%$ TdT enzyme in a humidified atmosphere at $37^{\circ} \mathrm{C}$ for $90 \mathrm{~min}$. Subsequently, the cells were stained with DAPI (Sigma-Aldrich; Merck KGaA) at $4^{\circ} \mathrm{C}$ for $10 \mathrm{~min}$. Finally, the cells were observed under a fluorescence microscope (magnification, x200). An average of 10 random fields with 100-200 nuclei per field was analyzed. The number of TUNEL-positive nuclei (green fluorescence) was expressed as the percentage of total nuclei (blue fluorescence).

Flow cytometry. The effects of miR-134-3p on the cell cycle were measured by flow cytometry as previously described (10). Briefly, the transfected cells were harvested, washed and 
suspended in $250 \mu 1$ of ice-cold PBS. Subsequently, $750 \mu 1$ of $100 \%$ ice-cold ethanol were added and slowly mixed with the cell suspensions for $\sim 8 \mathrm{~h}$ for fixation on ice. Subsequently, the cells were washed with PBS and incubated with RNase $(50 \mu \mathrm{g} / \mathrm{ml})$ and propidium iodide $(50 \mu \mathrm{g} / \mathrm{ml}$, Thermo Fisher Scientific, Inc.) for $30 \mathrm{~min}$ on ice. Finally, the cell cycle was detected using a Flow Cytometry System (BD Accuri C6; BD Biosciences) and the relative ratios of $G 0 / \mathrm{G}_{1}, \mathrm{~S}$ and $\mathrm{G}_{2} / \mathrm{M}$ phases were analyzed using FlowJo VX software (v10.0.7; FlowJo LLC).

Wound healing assay. Wound healing assay was performed to examine the migration of the cells as previously described (22). Briefly, serum-starved SKOV-3 and OVCAR-3 cells were seeded in each well of a 6-well plate, and a wound was created using a 100- $\mu$ l pipette tip. Following culture for a further $48 \mathrm{~h}$ at $37^{\circ} \mathrm{C}$, the wound recovery area was evaluated using a light microscope (Nikon Corporation; magnification, x200).

Transwell chamber assay. To explore the migration and invasion of SKOV-3 and OVCAR-3 cells, the Transwell chamber assay was performed as follows. The transfected SKOV-3 and OVCAR-3 cells $\left(3 \times 10^{4}\right)$ were seeded into the upper chamber of a Matrigel pre-coated Transwell chamber and cultured in their respective serum-free medium. Complete culture medium was added to the lower chamber. Following $48 \mathrm{~h}$ of culture at $37^{\circ} \mathrm{C}$, the cells in the lower chamber were fixed with $4 \%$ paraformaldehyde for $30 \mathrm{~min}$ at $4^{\circ} \mathrm{C}$ and stained with $5 \%$ crystal violet at $4^{\circ} \mathrm{C}$ for $30 \mathrm{~min}$. The number of cells passing through the Matrigel matrix were counted and photographed under a light microscope (magnification, x200).

Dual-Luciferase reporter assay. Firstly, the starBase database (http://starbase.sysu.edu.cn/) was used for prediction of miR-134-3p target genes. $\mathrm{R}<-0.1$ and $\mathrm{P}<0.05$ were set as the cut-off criteria for identifying the significant miRNA/gene pairs. One of the identified target genes was FEN1. Subsequently, FEN1-wild-type (WT) or FEN1-mutant (MUT) reporter plasmids were synthesized from Shanghai GenePharma Co., Ltd. Briefly, SKOV-3 and OVCAR-3 cells were co-transfected with $0.24 \mu \mathrm{g}$ of the FEN1-WT or FEN1-MUT reporter plasmids together with $40 \mathrm{nM}$ of miR-134-3p mimic or NC mimic using the cell electroporation system operator H1, as aforementioned. Additionally, $0.05 \mu \mathrm{g}$ of Renilla luciferase expression plasmid (Promega Corporation) was transfected into the cells as a reference control. The transfected cells were seeded into 24 -well plates for $36 \mathrm{~h}$ of culture at $37^{\circ} \mathrm{C}$. Finally, firefly and Renilla luciferase activities in the cells were measured using the Dual-Luciferase Reporter Assay System (Promega Corporation). The ratio of firefly and Renilla luciferase activities was calculated as the relative luciferase activity.

Western blot analysis. Cells were harvested using RIPA lysis buffer, Total proteins (40 $\mu \mathrm{g} /$ lane) were separated via $12 \%$ SDS-PAGE and were transferred onto PVDF membranes (EMD Millipore) using a MiniGenie blotting system (Bio-Rad Laboratories, Inc.). The membranes were then blocked with TBS-Tween (TBST; $0.1 \%$ Tween-20) containing 1\% skim milk powder at room temperature for $1 \mathrm{~h}$, and then incubated with rabbit monoclonal primary antibodies against human p21
(1:1,000; cat. no. 2947S), cyclooxygenase-2 (Cox-2; 1:1,000; cat. no. 12282T), matrix metalloproteinase (MMP)2 (1:1,000; cat. no. 40994S), MMP9 (1:1,000; cat. no. 13667S), cyclin D1 (1:1,000; cat. no. 55506S), CDK2 (1:1,000; cat. no. 2546S), Bax (1:1,000; cat. no. 5023S), cleaved caspase-3 (1:1,000; cat. no. 9654S), cleaved caspase-9 (1:1,000; cat. no. 20750S), Bcl-2 (1:1,000; cat. no. 4223S), $\beta$-actin (1:1,000; cat. no. 4970T) and FEN1 (1:2,000; cat. no. 82354S) (all from Cell Signaling Technology, Inc.) at $4{ }^{\circ} \mathrm{C}$ overnight. After washing with TBST, the membranes were incubated with goat anti-rabbit secondary antibodies (1:10,000; cat. no. 14708S; Cell Signaling Technology, Inc.) for $1 \mathrm{~h}$ at room temperature, followed by visualization with an enhanced chemiluminescence system (BeyoECL Plus; Beyotime Institute of Biotechnology). The protein bands were quantified using ImageJ software $(\mathrm{v} 1.48 \mathrm{u}$; National Institutes of Health).

Statistical analysis. All experiments were performed in triplicate independently. Quantitative data are presented as the mean \pm SD. GraphPad Prism (v6.01; GraphPad Software, Inc.) was used to perform the statistical analyses. The unpaired Student's t-test was used to compare differences between two groups, while one-way ANOVA followed Bonferroni's posthoc test was used when comparing $>2$ groups. $\mathrm{P}<0.05$ was considered to indicate a statistically significant difference.

\section{Results}

miR-134-3p inhibits the proliferation of OC cells. To confirm whether miR-134-3p served a role in the progression of OC, its expression levels were compared between human ovarian cancer cell lines (SKOV-3, A2780 and OVCAR-3) and a normal ovarian epithelial cell line (ISOE-80) by RT-qPCR. The results revealed that the expression levels of miR-134-3p in the human OC cell lines were significantly lower than those in the normal ovarian cell line $(\mathrm{P}<0.01$; Fig. $1 \mathrm{~A})$. As miR-134-3p expression was lowest in the SKOV-3 and OVCAR-3 cells, these two cell lines were selected for further analyses. Subsequently, to evaluate the effects of miR-134-3p on the progression ofOC, SKOV-3 and OVCAR-3 cells were transfected with miR-134-3p mimic or NC mimic. The RT-qPCR results revealed that miR-134-3p mimic significantly upregulated miR-134-3p expression in SKOV-3 and OVCAR-3 cells $(\mathrm{P}<0.01$; Fig. $1 \mathrm{~B})$. In addition, the CCK- 8 assay revealed that miR-134-3p mimic significantly decreased the viability of SKOV-3 and OVCAR-3 cells after $72 \mathrm{~h}(\mathrm{P}<0.05$; Fig. 1C). Colony formation assay demonstrated that miR-134-3p mimic significantly decreased the proliferation of SKOV-3 and OVCAR-3 cells ( $\mathrm{P}<0.01$; Fig. 1D). Overall, these data demonstrated that miR-134-3p inhibited the proliferation of $\mathrm{OC}$ cells.

miR-134-3p facilitates cell apoptosis and induces cell cycle arrest at the $G_{0} / G_{1}$ phase in SKOV-3 and OVCAR-3 cells. The regulation of apoptosis and cell cycle arrest serves an important role in cell proliferation $(12,13)$. In the present study, to reveal the mechanisms of miR-134-3p in OC cell proliferation, TUNEL and flow cytometry assays were performed to examine the effects of miR-134-3p overexpression on cell apoptosis and the cell cycle. First, the results of the TUNEL 

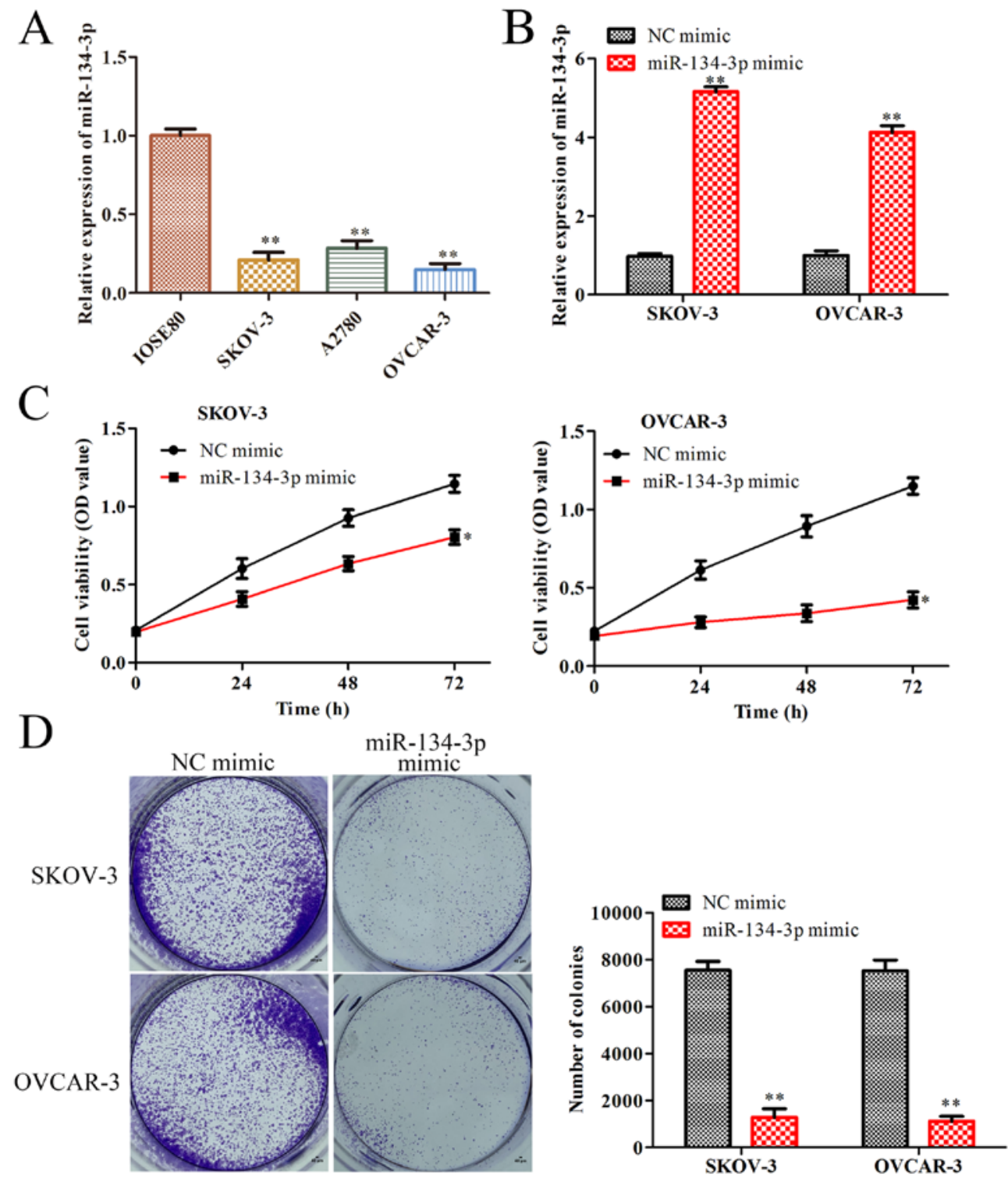

Figure 1. miR-134-3p inhibits the proliferation of SKOV-3 and OVCAR-3 cells. SKOV-3 and OVCAR-3 cells were transfected with miR-134-3p mimic or NC mimic. (A) Reverse transcription-quantitative PCR analysis was used to determine miR-134-3p expression in SKOV-3, A2780, OVCAR-3 and ISOE-80 cells. (B) miR-134-3p expression in SKOV-3 and OVCAR-3 cells at $48 \mathrm{~h}$ following transfection. (C) Cell Counting Kit-8 was performed to measure cell viability (D) Colony formation assays were performed to measure cell proliferation (magnification, $\mathrm{x} 40)$. The data are presented as the mean $\pm \mathrm{SD}(\mathrm{n}=3)$. ${ }^{*} \mathrm{P}<0.05$ and ${ }^{* *} \mathrm{P}<0.01$ vs. IOSE80 or NC mimic. miR, microRNA; NC, negative control; OD, optical density.

assay indicated that the miR-134-3p mimic significantly facilitated SKOV-3 and OVCAR-3 cell apoptosis ( $\mathrm{P}<0.01$; Fig. 2A). Furthermore, the analysis of cell apoptosis-associated proteins by western blot analysis revealed that the miR-134-3p mimic significantly increased the expression levels of Bax, cleaved caspase- 3 and cleaved caspase- $9(\mathrm{P}<0.01)$, and significantly decreased those of Bcl-2 in SKOV-3 and OVCAR-3 cells $(\mathrm{P}<0.05$; Fig. 2B). Subsequently, the data from the flow cytometry indicated that the miR-134-3p mimic significantly increased the percentage of cells at the $G_{0} / G_{1}$ phase, while decreasing the percentage of cells at the $S$ and $G_{2} / M$ phases in both SKOV-3 and OVCAR-3 cells ( $\mathrm{P}<0.01$; Fig. 2C). Finally, western blot analysis was performed to assess the expression levels of cell cycle-associated proteins. The results revealed that miR-134-3p mimic significantly decreased the protein expression levels of cyclin D1 and CDK2, while increasing the protein expression levels of $\mathrm{p} 21$ ( $\mathrm{P}<0.05$ and $\mathrm{P}<0.01$, Fig. 2D).
Overall, these results indicated miR-134-3p inhibited cell proliferation by facilitating cell apoptosis and inducing cell cycle arrest at the $\mathrm{G}_{0} / \mathrm{G}_{1}$ phase in $\mathrm{OC}$.

miR-134-3p inhibits the migration of SKOV-3 and OVCAR-3 cells. To further investigate the effects of miR-134-3p on the migration and invasion of OC cells, wound healing and Transwell assays were performed. The results of the wound healing assay indicated that the miR-134-3p mimic significantly decreased the wound closure ability of SKOV-3 and OVCAR-3 cells $(\mathrm{P}<0.01$; Fig. 3A), suggesting that miR-134-3p inhibited cell migration. Similarly, Transwell assays demonstrated that the miR-134-3p mimic significantly inhibited the migration and invasion of SKOV-3 and OVCAR-3 cells $(\mathrm{P}<0.01$; Fig. 3B). At the molecular level, western blot analysis revealed that the miR-134-3p mimic significantly decreased the expression levels of the migration-associated proteins MMP2, 

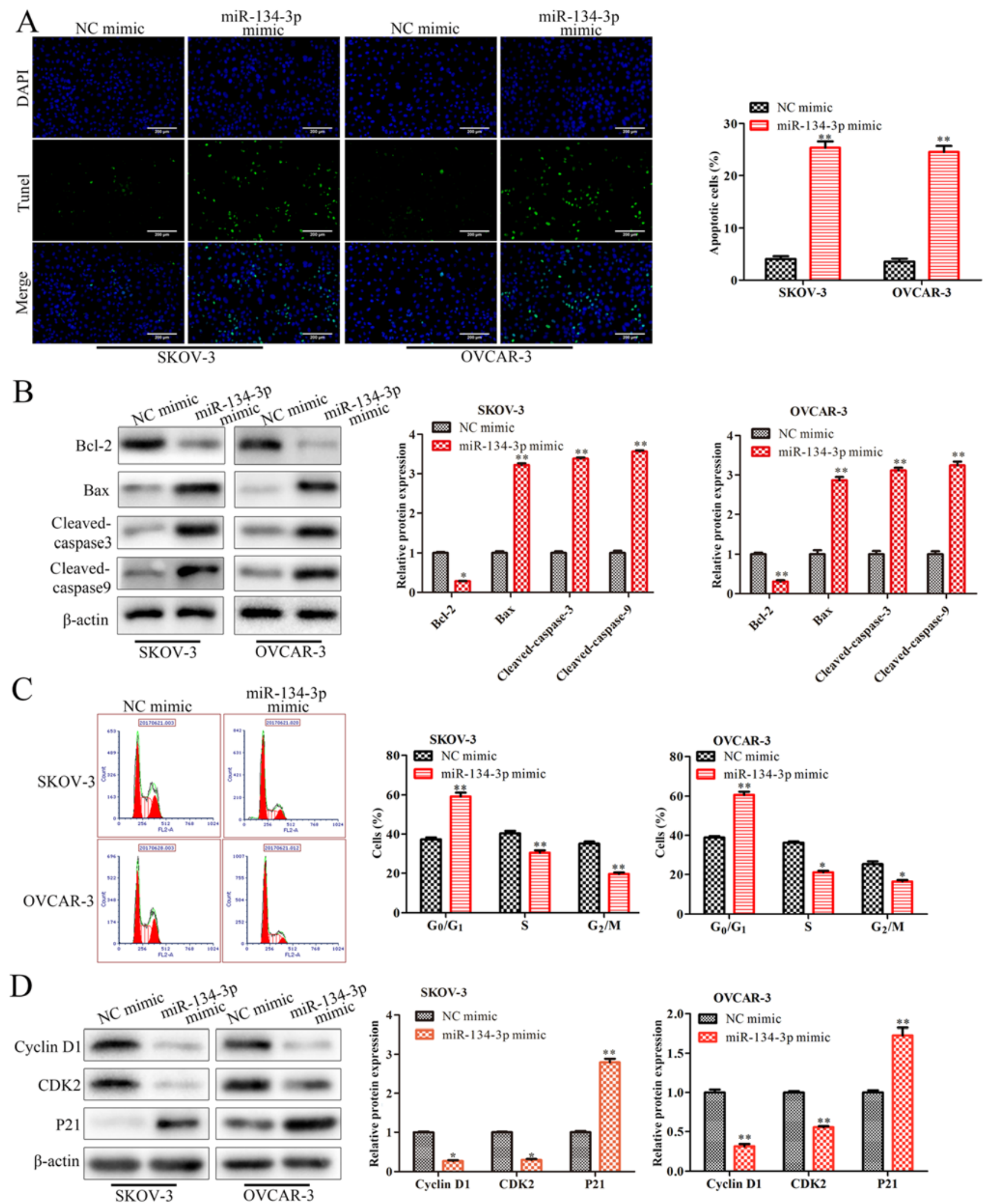

Figure 2. miR-134-3p facilitates cell apoptosis and induces cell cycle arrest at the $\mathrm{G}_{0} / \mathrm{G}_{1}$ phase in SKOV-3 and OVCAR-3 cells. SKOV-3 and OVCAR-3 cells were transfected with miR-134-3p mimic or NC mimic. (A) TUNEL assay was performed to assess cell apoptosis (scale bar, $200 \mu \mathrm{m}$ ). (B) Western blot analysis was used to assess the protein expression levels of Blc-2, Bax, cleaved caspase-3 and cleaved caspase-9. (C) Flow cytometry assay was performed to assess the cell cycle. (D) Western blot analysis was used to assess the protein expression levels of cyclin D1, CDK2 and p21. The data are presented as the mean \pm SD $(\mathrm{n}=3) .{ }^{*} \mathrm{P}<0.05$ and $^{* * *} \mathrm{P}<0.01$ vs. NC mimic. PCNA, proliferating cell nuclear antigen; miR, microRNA; NC, negative control.

MMP9 and Cox-2 ( $\mathrm{P}<0.01$; Fig. 3C). Collectively, these results demonstrated that miR-134-3p decreased the migratory and invasive abilities of OC cells.
miR-134-3p inhibits FEN1 expression by directly binding to the 3'-UTR of FEN1. To explore the detailed mechanisms responsible for the regulatory effects of miR-134-3p on OC 
A

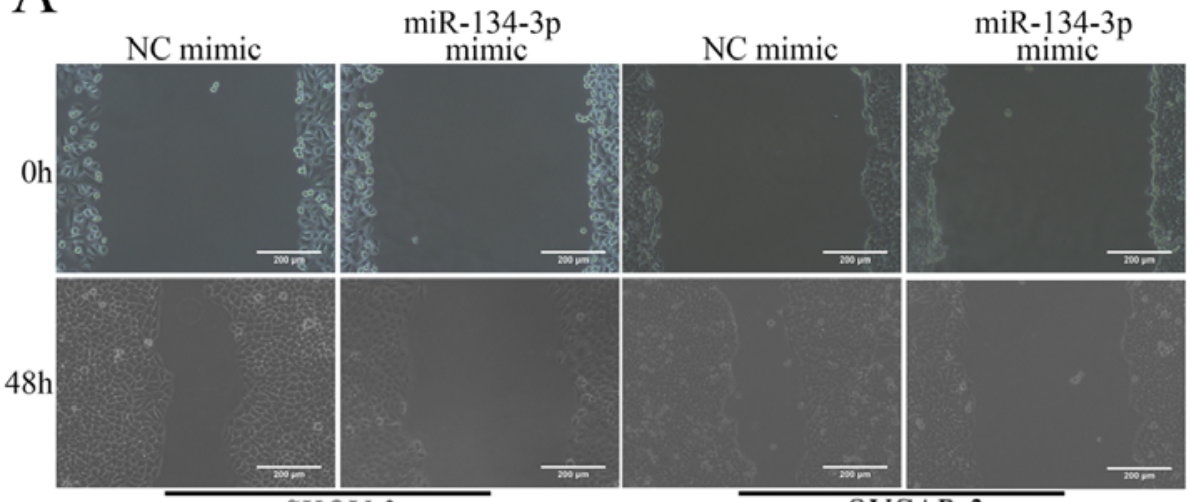

SKOV-3

OVCAR-3

B

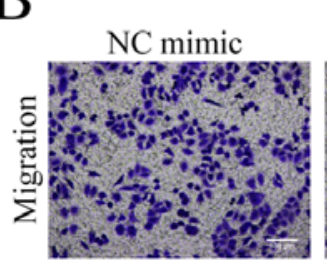

miR-134-3p mimic
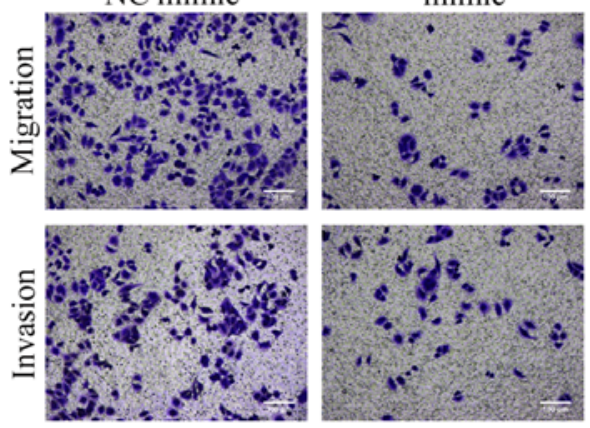

SKOV-3

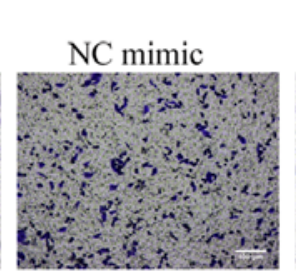

miR-134-3p mimic
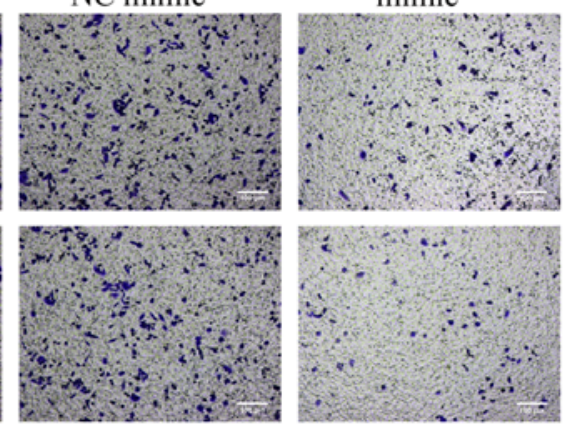

OVCAR-3

C
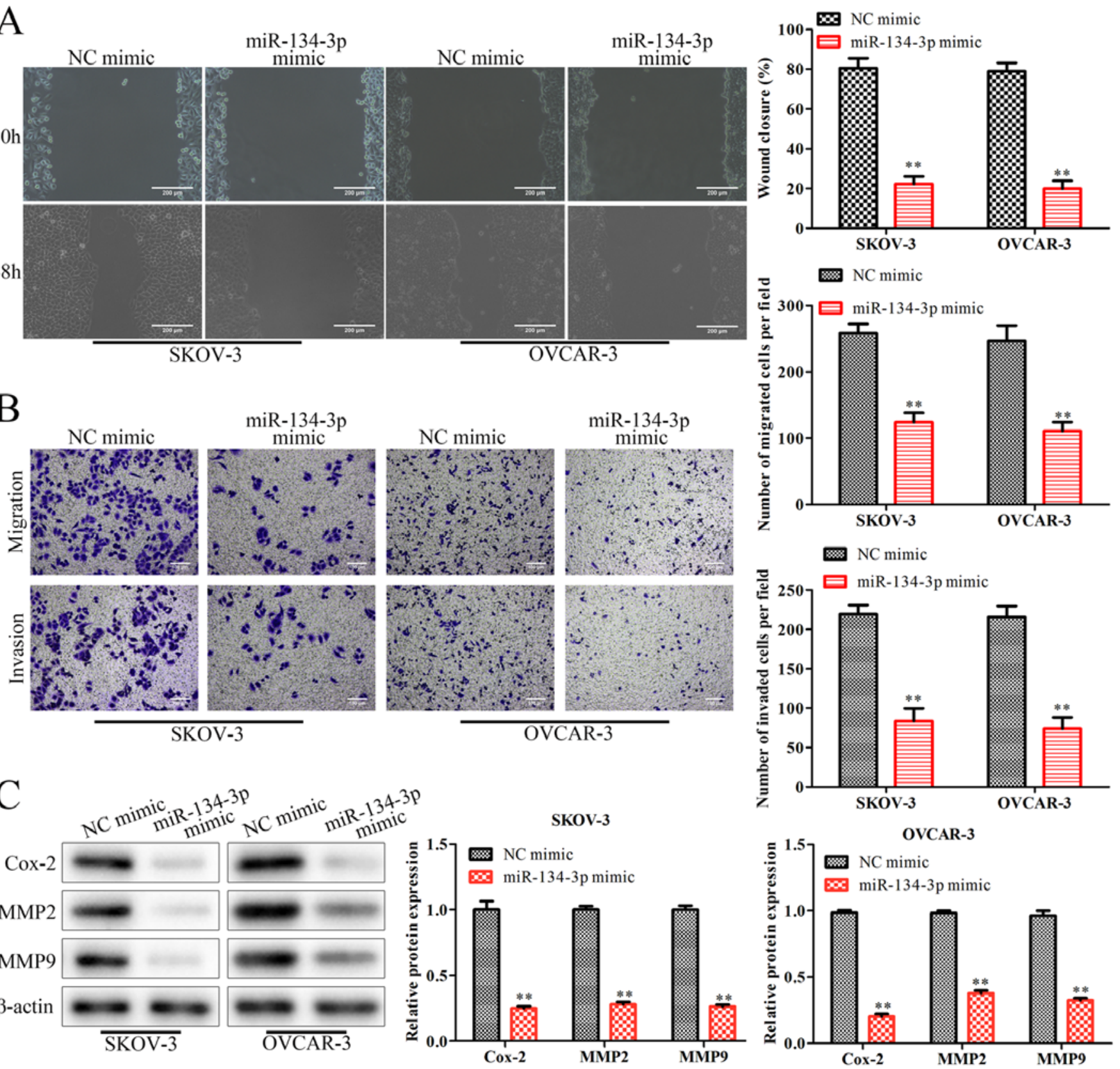

Figure 3. miR-134-3p inhibits cell migration of SKOV-3 and OVCAR-3 cells. SKOV-3 and OVCAR-3 cells were transfected with miR-134-3p mimic or inhibitor. (A) Wound healing assay was employed to assess the migration of the cells (scale bar, $200 \mu \mathrm{m}$ ). (B) Transwell assay was performed to assess cell migration and invasion (scale bar, $100 \mu \mathrm{m}$ ). (C) Western blot analysis was performed to assess the expression levels of Cox-2, MMP2 and MMP9 proteins. The data are presented as the mean $\pm \mathrm{SD}(\mathrm{n}=3) .{ }^{* *} \mathrm{P}<0.01$ vs. NC mimic. miR, microRNA; NC, negative control; MMP, matrix metalloproteinase; Cox-2, cyclooxygenase 2 .

progression, starBase was used to screen for miR-134-3p target genes, revealing that FEN1 may be one of the target genes of miR-134-3p (Fig. 4A). Subsequently, a Dual-Luciferase reporter assay was performed to verify this interaction. The results indicated that the miR-134-3p mimic significantly downregulated the luciferase activity of FEN1-WT, but not that of FEN1-MUT ( $<<0.01$; Fig. 4B). The RT-qPCR results (Fig. 4C) and western blot analysis (Fig. 4D) demonstrated that the miR-134-3p mimic significantly downregulated the mRNA and protein expression levels of FEN1 $(\mathrm{P}<0.01)$. These data confirmed that miR-134-3p inhibited the expression levels of the FEN1 gene by directly binding to the 3'-UTR of FEN1 in $\mathrm{OC}$ cells.
Overexpression of FEN1 reverses the effects of miR-134-3p on SKOV-3 and OVCAR-3 cells. To confirm the association between miR-134-3p and FEN1, miR-134-3p mimic or NC mimic were co-transfected with Lv-FEN1 or Lv-NC into SKOV-3 and OVCAR-3 cells. RT-qPCR revealed that Lv-FEN1 significantly increased the expression levels of FEN1 in SKOV-3 and OVCAR-3 cells ( $\mathrm{P}<0.01$; Fig. 5A). To examine the mediatory effects of FEN1 on OC cells, a CCK-8 assay was conducted, revealing that co-transfection with the miR-134-3p mimic + Lv-FEN1 or miR-134-3p mimic + Lv-NC significantly decreased the viability of SKOV-3 and OVCAR-3 cells compared with that of the cells co-transfected with NC mimic + Lv-NC $(\mathrm{P}<0.05$; Fig. 5B). Additionally, the viability of 
A

$\begin{array}{|lc|}\text { FEN1 WT } & \text { 5' } \text {-UUAAUGGCAAGAAGGCCACAGAG-3' } \\ \text { I l l l l l } \\ \text { Hsa-miR-134-3p } & 3 \text { '-AACCACUGAUCCACCGGGUGUCC -5' } \\ & \\ \text { FEN1 Mut } & 5 \text { '-UUAAUGGCAAGAAGGGGUUUCAG -3' }\end{array}$

B

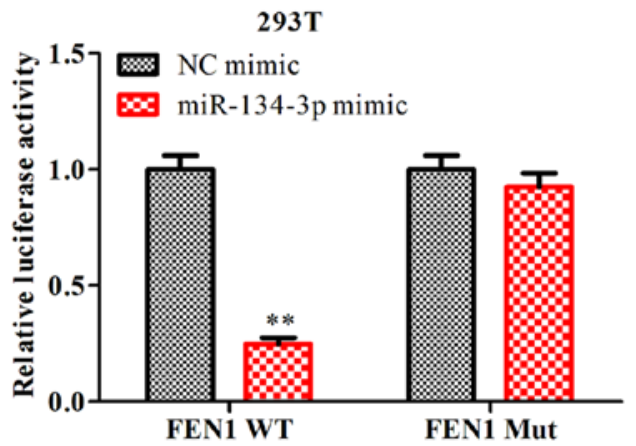

$\mathrm{D}$

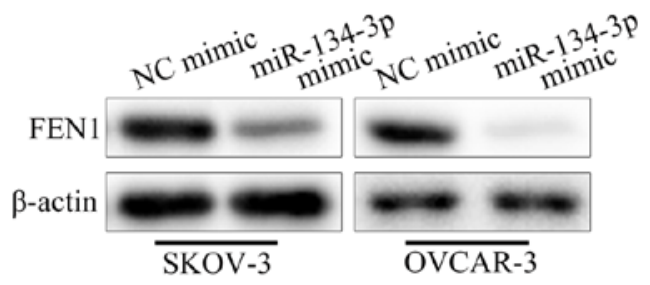

$\mathrm{C}$
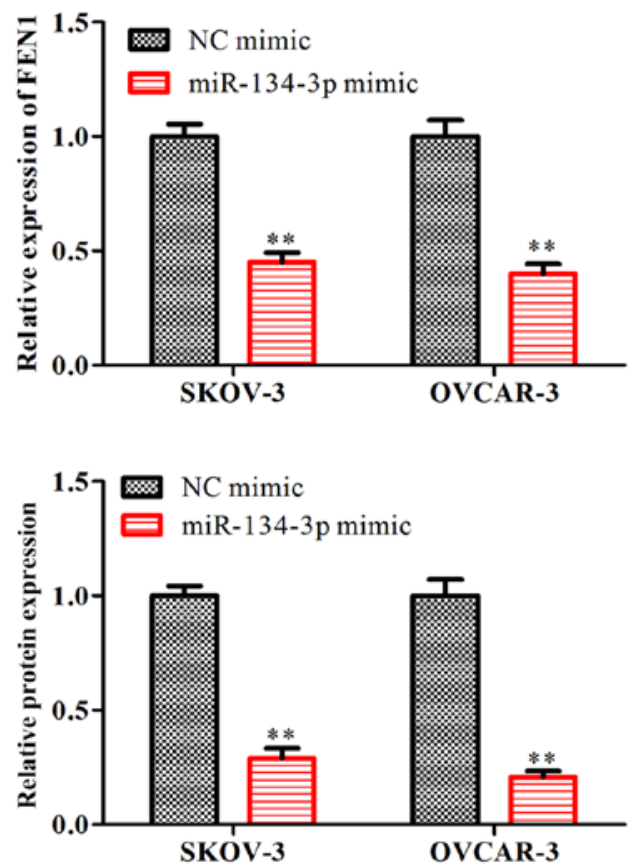

Figure 4. FEN1 serves as a direct miR-134-3p target in SKOV-3 and OVCAR-3 cells. (A) The putative binding site between FEN1 and miR-134-3p was predicted by starBase. (B) Dual-luciferase reporter assay was performed to reveal the association between miR-134-3p and FEN1. (C) Reverse transcriptionquantitative PCR was performed to assess the mRNA expression of FEN1. (D) Western blot analysis was employed to assess the protein expression levels of FEN1 in SKOV-3 and OVCAR-3 cells transfected with miR-134-3p mimic. The data are presented as the mean $\pm \mathrm{SD}(\mathrm{n}=3)$. ${ }^{* * *} \mathrm{P}<0.01 \mathrm{vs}$. NC mimic. miR, microRNA; NC, negative control; FEN1, flap structure-specific endonuclease 1; WT, wild-type; MUT, mutant.

SKOV-3 and OVCAR-3 cells co-transfected with miR-134-3p mimic + Lv-FEN1 was significantly higher than that of cells transfected with miR-134-3p mimic + Lv-NC (Fig. 5B). Colony formation assay revealed that, compared with Lv-NC, Lv-FEN1 significantly promoted the colony formation ability of SKOV-3 and OVCAR-3 cells transfected with miR-134-3p mimic $(\mathrm{P}<0.05$; Fig. $5 \mathrm{C})$. Moreover, TUNEL assay indicated that Lv-FEN1 significantly inhibited the apoptosis of miR-134-3p mimic-transfected SKOV-3 and OVCAR-3 cells compared with Lv-NC ( $\mathrm{P}<0.01$; Fig. 5D). Finally, Transwell assay revealed that Lv-FEN1 significantly increased the invasion of miR-134-3p mimic-transfected SKOV-3 and OVCAR-3 cells compared with Lv-NC ( $\mathrm{P}<0.01$; Fig. 5E). Overall, these results suggested that the overexpression of FEN1 partially reverses the effects of miR-134-3p overexpression on OC.

\section{Discussion}

This study revealed that the expression levels of miR-134-3p were decreased in OC cells compared with in normal ovarian cells. Functional and mechanical experiments further revealed that miR-134-3p inhibited the proliferation, migration and invasion of SKOV-3 and OVCAR-3 cells by downregulating
FEN1 expression. These preliminary experiments indicated that miR-134-3p may be a promising candidate for use in OC therapy.

A previous study has demonstrated that miR-134 serves crucial roles in the onset, progression and metastasis of various types of human cancer, such as lung cancer, glioma, breast cancer and colorectal cancer, by inhibiting the translation of target mRNAs (23). Furthermore, miR-134 has been reported to suppress the migration and invasion of non-small cell lung cancer by targeting integrin subunit $\beta 1$ (24). miR-134 has been identified to target programmed cell death protein 7 to decrease E-cadherin expression and enhance oral cancer progression (25). In addition, miR-134 has been reported to function as a novel potential inhibitor of human OC cell proliferation and cell cycle progression (26). The present study demonstrated that upregulating miR-134-3p expression inhibited the proliferation of SKOV-3 and OVCAR-3 cells by facilitating cell apoptosis and arresting the cell cycle. The current results are in accordance with those of a previous study demonstrating that downregulated miR-134 expression contributes to paclitaxel resistance in human OC cells (27). Additionally, the present western blot analysis results indicated that the overexpression of miR-134-3p decreased the protein 
A

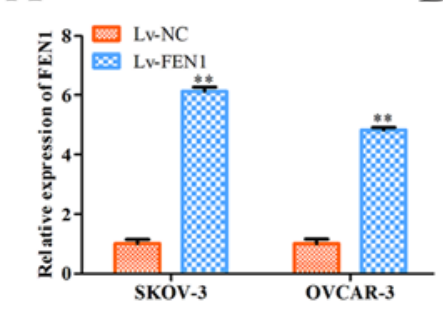

C $\underset{+\mathrm{LV}-\mathrm{NC}}{\mathrm{NC} \text { mimic }}$

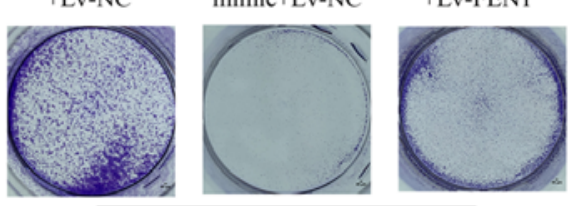

SKOV-3

D $\underset{+\mathrm{L} v-\mathrm{NC}}{\mathrm{NC} \text { mimic }}$

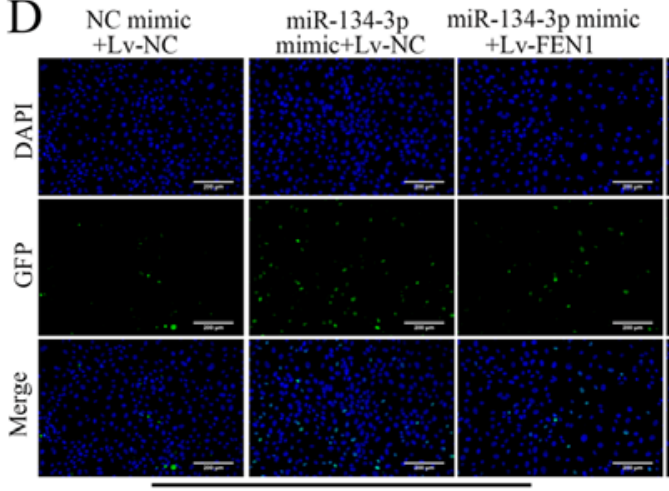

SKOV-3

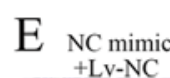

B

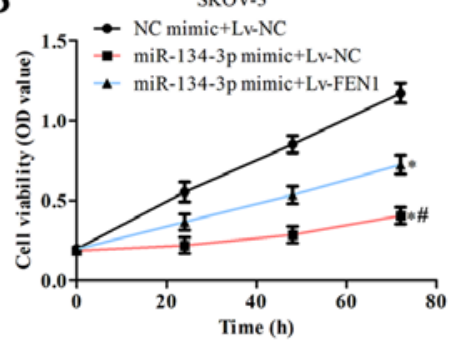

OVCAR-3

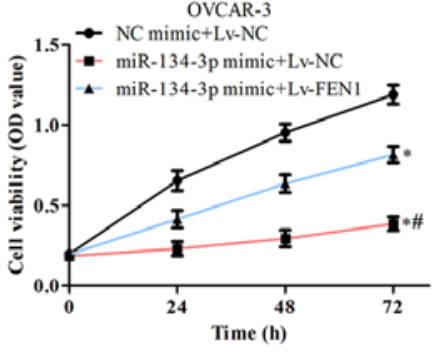

$\mathrm{NC}$ mimic $+\mathrm{Lv}-\mathrm{NC}$

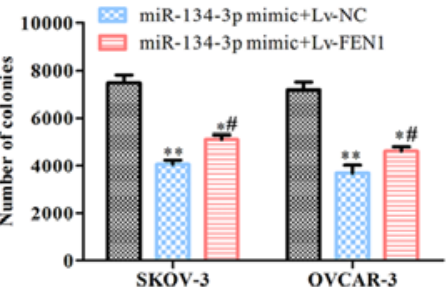

OVCAR-3

miR-134-3p miR-134-3p mimic

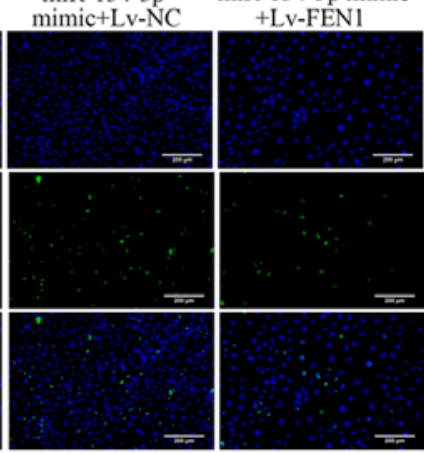

OVCAR-3

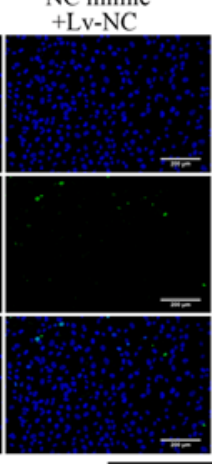

OVCAR-3 miR-134-3p
mimic+Lv-NC
+Lv-FEN1
+L-3p mimic
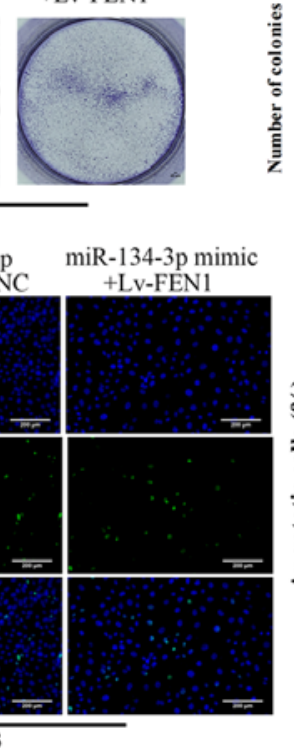

NC mimic + Lve $-\mathrm{NC}$

miR-134-3p mimic +Lv-NC $\left.{ }^{15}\right]$ miR-134-3p mimic +Lv-FENI
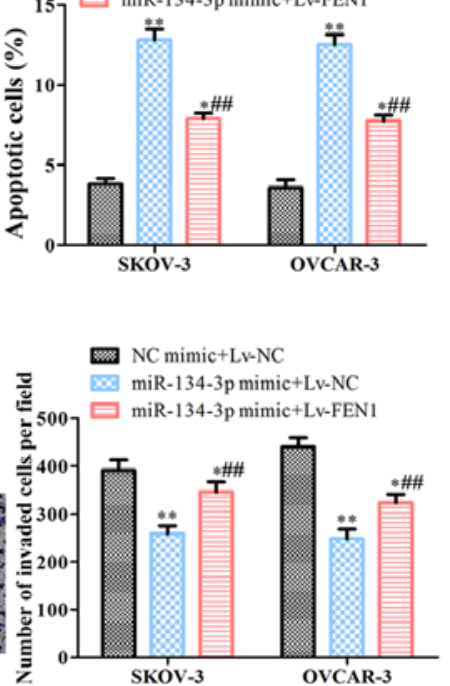

Figure 5. Overexpression of FEN1 reverses the effects of miR-134-3p on SKOV-3 and OVCAR-3 cells. SKOV-3 and OVCAR-3 cells were co-transfected with miR-134-3p mimic or NC mimic along with Lv-FEN1 or Lv-NC, respectively. (A) Reverse transcription-quantitative PCR was performed to assess FEN1 expression. (B) Cell Counting Kit- 8 was performed to assess cell viability at $0,24,48$ and 72 h. (C) Colony formation was performed to assess colony formation of the cells (magnification, x40). (D) TUNEL (scale bar, $200 \mu \mathrm{m}$ ) and (E) Transwell assays (scale bar, 100 $\mu \mathrm{m}$ ) were performed to assess the apoptosis and invasion of the cells, respectively. The data are presented as the mean $\pm \mathrm{SD}(\mathrm{n}=3) .{ }^{*} \mathrm{P}<0.05$ and ${ }^{* *} \mathrm{P}<0.01$ vs. NC mimic+Lv-NC. ${ }^{\#} \mathrm{P}<0.05$ and ${ }^{\# \#} \mathrm{P}<0.01$ vs. miR-134-3p mimic+Lv-NC. miR, microRNA; NC, negative control; FEN1, flap structure-specific endonuclease 1; OD, optical density; Lv, lentiviral expression vector (pcDNA3.1).

expression levels of cyclin D1, CDK2 and PCNA. The expression levels of Cox-2, MMP2 and MMP9 were also decreased by the miR-134-3p mimic.

The prediction results by starBase revealed that FEN1 was the direct target of miR-134-3p. Furthermore, this interaction was validated by luciferase reporter assays, and it was demonstrated that miR-134-3p interacted directly with the 3'-UTR of FEN1. FEN1, also known as DNase IV, is the mammalian counterpart of the distinct 59 nuclease domain of Escherichia coli DNA polymerase I (28). FEN1 is directly involved in DNA replication and repair, interacting with the interdomain connector loop of PCNA (29). In cancer progression, FEN1 mediates osteosarcoma cell autophagy through the disruption of DNA damage repair processes (20). It has been demonstrated that FEN1 mediates the effects of miR-200a and promotes breast cancer cell proliferation via MET and
EGFR signaling (30). Moreover, miR-140 impedes DNA repair by targeting FEN1 and sensitizes breast cancer cells to the chemotherapeutic drug doxorubicin (19). It has been reported that FEN1 overexpression is associated with a high grade, high tumor stage and a poor survival in patients with ovarian epithelial cancer (31). The results of the present study indicated that miR-134-3p decreased the expression levels of FEN1 in SKOV-3 and OVCAR-3 cells. The overexpression of FEN1 reversed the effects of miR-134-3p on the proliferation, migration and invasion of SKOV-3 and OVCAR-3 cells.

The present study has some limitations. No comprehensive analysis of miR-134-3p expression in OC and normal ovarian tissues was performed. The current results only demonstrated the effects of miR-134-3p in OC cells in vitro. Future studies should focus on the expression levels of miR-134-3p in patients with different stages of OC. In addition, the effects 
of miR-134-3p/FEN1 in OC in vivo should be further investigated in future research.

In conclusion, the present study suggested that miR-134-3p may modulate FEN1 expression to inhibit the progression of OC. The present study provided the molecular mechanisms for understanding the role of miR-134-3p in the regulation of the proliferation and metastasis of $\mathrm{OC}$.

\section{Acknowledgements}

Not applicable.

\section{Funding}

The present study was supported by the Central South University Post-Graduate Independent Exploration and Innovation Project (grant no. 2017zzts382), the Central South University Graduate excellent course (grant no. 2014jpkc003), the Hunan Provincial Natural Science Foundation of China (grant no. 2015JJ2165) and the Central South University Fundamental Research Funds Special Funding (grant no. 165611031).

\section{Availability of data and materials}

The datasets used and/or analyzed during the current study are available from the corresponding author on reasonable request.

\section{Authors' contributions}

YY and MZ conceived and designed the study. MZ, HJ and QF performed the experiments. QC and YZ analyzed the data. MZ drafted the manuscript. YY and YZ edited and revised the manuscript. All authors have read and approved the final manuscript.

\section{Ethics approval and consent to participate}

Not applicable.

\section{Patient consent for publication}

Not applicable.

\section{Competing interests}

The authors declare that they have no competing interests.

\section{References}

1. Bray F, Ferlay J, Soerjomataram I, Siegel RL, Torre LA and Jemal A: Global cancer statistics 2018: GLOBOCAN estimates of incidence and mortality worldwide for 36 cancers in 185 countries. CA Cancer J Clin 68: 394-424, 2018.

2. Torre LA, Trabert B, DeSantis CE, Miller KD, Samimi G, Runowicz CD, Gaudet MM, Jemal A and Siegel RL: Ovarian cancer statistics, 2018. CA Cancer J Clin 68: 284-296, 2018.

3. Zhang ML, Peng P, Wu CX, Gong YM, Zhang SW, Chen WQ and Bao PP: [Report of breast cancer incidence and mortality in China registry regions, 2008-2012]. Zhonghua Zhong Liu Za Zhi 41: 315-320, 2019 (In Chinese).
4. Marth C, Reimer D and Zeimet AG: Front-line therapy of advanced epithelial ovarian cancer: standard treatment. Ann Oncol 28 (Suppl 8): viii36-viii39, 2019. https://doi.org/10.1093/ annonc/mdx450.

5. Bieg D, Sypniewski D, Nowak E and Bednarek I: MiR-424-3p suppresses galectin-3 expression and sensitizes ovarian cancer cells to cisplatin. Arch Gynecol Obstet 299: 1077-1087, 2019.

6. Yang B, Sun L and Liang L: MiRNA-802 suppresses proliferation and migration of epithelial ovarian cancer cells by targeting YWHAZ. J Ovarian Res 12: 100, 2019.

7. Ding Y, Fang Q, Li Y and Wang Y: Amplification of lncRNA PVT1 promotes ovarian cancer proliferation by binding to miR-140. Mamm Genome 30: 217-225, 2019.

8. Fan B, Chen LP, Yuan YH, Xiao HN, Lv XS and Xia ZY: MiR-15a-3p suppresses the growth and metastasis of ovarian cancer cell by targeting Twist1. Eur Rev Med Pharmacol Sci 23: 1934-1946, 2019.

9. Zuo Y, Zheng W, Liu J, Tang Q, Wang SS and Yang XS: MiR-34a-5p/PD-L1 axis regulates cisplatin chemoresistance of ovarian cancer cells. Neoplasma 67: 93-101, 2020.

10. Zhang X, Xin G and Sun D: Serum exosomal miR-328, miR-575, miR-134 and miR-671-5p as potential biomarkers for the diagnosis of Kawasaki disease and the prediction of therapeutic outcomes of intravenous immunoglobulin therapy. Exp Ther Med 16: 2420-2432, 2018.

11. Chen CL, Zhang L, Jiao YR, Zhou Y, Ge QF, Li PC, Sun XJ and Lv Z: miR-134 inhibits osteosarcoma cell invasion and metastasis through targeting MMP1 and MMP3 in vitro and in vivo. FEBS Lett 593: 1089-1101, 2019.

12. Qin Q, Wei F, Zhang J, Wang X and Li B: miR-134 inhibits non-small cell lung cancer growth by targeting the epidermal growth factor receptor. J Cell Mol Med 20: 1974-1983, 2016.

13. Qi A, Han J, Jia F and Liu C: miR-3175 and miR-134 affect proliferation, invasion and apoptosis of glioma cells through PI3K/AKT signaling pathway. J BUON 24: 2465-2474, 2019.

14. Ma Z, Li K, Chen P, Pan Q, Li X and Zhao G: MiR-134, mediated by IRF1, suppresses tumorigenesis and progression by targeting VEGFA and MYCN in osteosarcoma. Anticancer Agents Med Chem 20: 1197-1208, 2020.

15. Greene AL, Snipe JR, Gordenin DA and Resnick MA: Functional analysis of human FEN1 in Saccharomyces cerevisiae and its role in genome stability. Hum Mol Genet 8: 2263-2273, 1999.

16. Gary R, Park MS, Nolan JP, Cornelius HL, Kozyreva OG, Tran HT, Lobachev KS, Resnick MA and Gordenin DA: A novel role in DNA metabolism for the binding of Fen1/Rad27 to PCNA and implications for genetic risk. Mol Cell Biol 19: 5373-5382, 1999.

17. Gomes XV and Burgers PM: Two modes of FEN1 binding to PCNA regulated by DNA. EMBO J 19: 3811-3821, 2000.

18. Nikolova T, Christmann M and Kaina B: FEN1 is overexpressed in testis, lung and brain tumors. Anticancer Res 29: 2453-2459, 2009.

19. Lu X, Liu R, Wang M, Kumar AK, Pan F, He L, Hu Z and Guo Z: MicroRNA-140 impedes DNA repair by targeting FEN1 and enhances chemotherapeutic response in breast cancer. Oncogene 39: 234-247, 2020.

20. 20. Dong S, Xiao Y, Ma X, He W, Kang J, Peng Z, Wang L and Li Z: miR-193b Increases the chemosensitivity of osteosarcoma cells by promoting FEN1-mediated autophagy. OncoTargets Ther 12: 10089-10098, 2019.

21. Livak KJ and Schmittgen TD: Analysis of relative gene expression data using real-time quantitative PCR and the 2(-Delta Delta C(T)) Method. Methods 25: 402-408, 2001.

22. Zhang YY, Li P, Zhu MZ, Guo Y and Yang J: LINC01308 accelerates the malignant progression of ovarian cancer by binding to miRNA-506. Eur Rev Med Pharmacol Sci 23: 3253-3260, 2019.

23. Pan JY, Zhang F, Sun CC, Li SJ, Li G, Gong FY, Bo T, He J, Hua RX, Hu WD, et al: miR-134: A human cancer suppressor? Mol Ther Nucleic Acids 6: 140-149, 2017.

24. Qin Q, Wei F, Zhang J and Li B: miR-134 suppresses the migration and invasion of non-small cell lung cancer by targeting ITGB1. Oncol Rep 37: 823-830, 2017.

25. Peng SY, Tu HF, Yang CC, Wu CH, Liu CJ, Chang KW and Lin SC: miR-134 targets PDCD7 to reduce E-cadherin expression and enhance oral cancer progression. Int J Cancer 143: 2892-2904, 2018

26. Chang C, Liu T, Huang Y, Qin W, Yang H and Chen J: MicroRNA$134-3 p$ is a novel potential inhibitor of human ovarian cancer stem cells by targeting RAB27A. Gene 605: 99-107, 2017. 
27. Shuang T, Wang M, Shi C, Zhou Y and Wang D: Down-regulated expression of miR-134 contributes to paclitaxel resistance in human ovarian cancer cells. FEBS Lett 589 (20 Pt B): 3154-3164, 2015.

28. Prasad R, Dianov GL, Bohr VA and Wilson SH: FEN1 stimulation of DNA polymerase beta mediates an excision step in mammalian long patch base excision repair. J Biol Chem 275: 4460-4466, 2000.

29. Storici F, Henneke G, Ferrari E, Gordenin DA, Hübscher U and Resnick MA: The flexible loop of human FEN1 endonuclease is required for flap cleavage during DNA replication and repair. EMBO J 21: 5930-5942, 2002.
30. Zeng X, Qu X, Zhao C, Xu L, Hou K, Liu Y, Zhang N, Feng J, Shi S, Zhang L, et al: FEN1 mediates miR-200a methylation and promotes breast cancer cell growth via MET and EGFR signaling. FASEB J 33: 10717-10730, 2019.

31. Abdel-Fatah TM, Russell R, Albarakati N, Maloney DJ, Dorjsuren D, Rueda OM, Moseley P, Mohan V, Sun H, Abbotts R, et al: Genomic and protein expression analysis reveals flap endonuclease 1 (FEN1) as a key biomarker in breast and ovarian cancer. Mol Oncol 8: 1326-1338, 2014.

(i) $(5)$ This work is licensed under a Creative Commons Attribution-NonCommercial-NoDerivatives 4.0 International (CC BY-NC-ND 4.0) License. 